\title{
Magneto-optical Stern-Gerlach forces and nonreciprocal torques on small particles
}

\author{
S. Edelstein, ${ }^{1}$ R. M. Abraham-Ekeroth,,${ }^{2,3}$ P. A. Serena, ${ }^{1}$ J. J. Sáenz, ${ }^{4,5}$ A. García-Martín,,${ }^{3, *}$ and M. I. Marqués $\oplus^{6, \dagger}$ \\ ${ }^{1}$ Instituto de Ciencia de Materiales de Madrid (ICMM-CSIC), Campus de Cantoblanco, 28049 Madrid, Spain \\ ${ }^{2}$ Instituto de Física Arroyo Seco, Universidad Nacional del Centro de la Provincia de Buenos Aires, Pinto 399, 7000 Tandil, Argentina \\ ${ }^{3}$ Instituto de Micro y Nanotecnología IMN-CNM, CSIC, CEI UAM+CSIC, Isaac Newton 8, Tres Cantos, 28760 Madrid, Spain \\ ${ }^{4}$ Donostia International Physics Center (DIPC), Paseo Manuel Lardizabal 4, 20018 Donostia-San Sebastian, Spain \\ ${ }^{5}$ IKERBASQUE, Basque Foundation for Science, 48013 Bilbao, Spain \\ ${ }^{6}$ Departamento de Física de Materiales, Condensed Matter Physics Center (IFIMAC) and Instituto Nicolás Cabrera, \\ Universidad Autónoma de Madrid, 28049 Madrid, Spain
}

(Received 13 May 2019; published 9 August 2019)

\begin{abstract}
In this paper we calculate the optical forces and torques caused by the presence of a sizable magneto-optical effect. We find a conservative force proportional to the gradient of the spin density of the light field and an extinction force proportional to the helicity of the light field. The conservative interaction allows for a spin-selective, magnetic field based Stern-Gerlach experiment, capable of differentiating between right and left circular polarizations. We also prove that by using a spinless linearly polarized plane wave, the magneto-optical effect allows for the existence of a permanent nonreciprocal torque, proportional to the intensity of the light field.
\end{abstract}

DOI: 10.1103/PhysRevResearch.1.013005

\section{INTRODUCTION}

The Stern-Gerlach experiment is one of the most representative examples of a measurement dealing with stateselective deflection in a field gradient. In this experiment, atoms are deflected depending on their magnetic moment by a nonconstant magnetic field. The splitting of an atomic beam in an optical field intensity gradient is often referred to as the optical Stern-Gerlach effect, first experimentally observed by Sleator and co-workers [1]. More recently, the discrimination of chiral molecules using optical forces has been proposed [2] and an experimental demonstration using optical helicity gradients has been reported [3]. In those optical analogs the electromagnetic radiation is always used as the splitting force. Our main goal here is to show that nonreciprocal optical forces can lead to an actual, counterintuitive, optical analog of the Stern-Gerlach effect: the photon-spin selective deflection of a light beam in a static magnetic field gradient.

There are many ways to split a beam of light in left and right circular polarizations based on standard circular filters. New alternative methods somehow related to Stern-Gerlachlike mechanisms are based on nonlinear coupling on pumped crystals with a transverse gradient, recently proposed to split spinorlike states of light [4], on scattering from chiral surfaces [5], or on electromagnetically induced transparency [6]. In the latter, the so-called dark polaritons, i.e., atom plus photon couplings with a nonzero magnetic moment depending on

\footnotetext{
*a.garcia.martin@csic.es

†manuel.marques@uam.es
}

Published by the American Physical Society under the terms of the Creative Commons Attribution 4.0 International license. Further distribution of this work must maintain attribution to the author(s) and the published article's title, journal citation, and DOI. the polarization of light, are created in atomic gases [7] and deflected by magnetic field gradients [8-10]. Our main goal here is to explore another way to extract information about the state of light (spin angular momentum and helicity) by using an external magnetic field to tune the optical forces on small particles via the magneto-optical effect (MOE).

After Ashkin's pioneering work of 1970 [11], driving, trapping, and shorting of nano- and micron-sized particles using electromagnetic fields have become common tools in physics and biology. Additionally, since the early days in the field of photonics, a key goal has been to be able to manipulate light-matter interactions using external means. Among all possibilities, one that offers high modulation speed and ease of action is the exploitation of the magneto-optical effect [12]. Indeed, the magneto-optical control of the resonant behavior in plasmonic systems, and the complementary enhancement of the magneto-optical response due to the same resonances, is behind the success of the field of magnetoplasmonics [13]. In the last few years, the standard magnetoplasmonic system exhibiting electric resonances has been extended to consider other situations, such us the exploitation of magnetic resonances [14-16], or even the control of thermal radiation and radiative heat transfer at room temperature [17-20].

In this paper we will merge optomechanics with the field of magneto-optics to analyze how optical forces and torques are tuned by the presence of an external magnetic field, and we will analyze how this modulation can be used to extract information about the spinning state of light using the classical Stern-Gerlach experiment.

\section{OPTICAL FORCE ON MAGNETO-OPTICAL PARTICLES}

We start by considering a particle made of isotropic material with permittivity $\varepsilon(\omega)$, in an otherwise homogeneous 
and isotropic medium with permittivity $\varepsilon_{h}$ and real refractive index $n_{h}=\sqrt{\varepsilon_{h}}$. For harmonic fields, the electric displacement $\mathbf{D}_{\text {in }}$ inside the particle is related to the electric field $\mathbf{E}_{\text {in }}$ through $\mathbf{D}_{\text {in }}=\varepsilon_{0} \varepsilon(\omega) \mathbf{E}_{i n}$. In the presence of a static external magnetic field $\mathbf{B}_{\text {ext }}$, the scalar permittivity becomes a tensor $\boldsymbol{\varepsilon}$ [with components $\varepsilon_{i j}\left(\mathbf{B}_{\text {ext }}\right)=\varepsilon_{j i}\left(-\mathbf{B}_{\text {ext }}\right)$ ] which, in the absence of absorption, must be Hermitian, $\varepsilon_{i j}\left(\mathbf{B}_{\text {ext }}\right)=$ $\varepsilon_{i j}^{*}\left(\mathbf{B}_{\text {ext }}\right)$. Assuming a small spherical particle of radius $R$, an external monochromatic field $\mathbf{E}$ induces an electric dipole $\mathbf{p}=\varepsilon_{0} \varepsilon_{h} \boldsymbol{\alpha} \mathbf{E}$, where $\boldsymbol{\alpha}(\omega)$ is the polarizability tensor given by $[21]$

$$
\begin{gathered}
\boldsymbol{\alpha}=\left(\boldsymbol{\alpha}_{0}^{-1}-\frac{i k^{3}}{6 \pi}\right)^{-1}, \\
\boldsymbol{\alpha}_{0}=3 V\left[\boldsymbol{\varepsilon}\left(\mathbf{B}_{\mathrm{ext}}\right)-\varepsilon_{h} \mathbf{I}\right]\left[\boldsymbol{\varepsilon}\left(\mathbf{B}_{\mathrm{ext}}\right)+2 \varepsilon_{h} \mathbf{I}\right]^{-1},
\end{gathered}
$$

where $k=n_{h} \omega / c$ is the wave number, $V=4 \pi R^{3} / 3$ is the particle volume, and $\mathbf{I}$ the unit tensor.

We will consider that the magneto-optical (MO) response is small, thus the electric displacement inside the particle, at lowest order in the external magnetic field (linear regime), is given by $[22,23]$

$$
\begin{aligned}
\mathbf{D}_{i n} & =\varepsilon_{0} \varepsilon \mathbf{E}_{i n}-i \varepsilon_{0} \frac{f(\omega) B_{\mathrm{ext}}}{\omega}\left(\begin{array}{ccc}
0 & b_{z} & -b_{y} \\
-b_{z} & 0 & b_{x} \\
b_{y} & -b_{x} & 0
\end{array}\right) \mathbf{E}_{i n} \\
& =\varepsilon_{0}\left(\varepsilon \mathbf{E}_{i n}-i \frac{f(\omega) B_{\mathrm{ext}}}{\omega} \mathbf{E}_{i n} \times \hat{\boldsymbol{b}}\right),
\end{aligned}
$$

where $\hat{\boldsymbol{b}}$ is a unit vector $\left(\mathbf{B}_{\mathrm{ext}}=B_{\mathrm{ext}} \hat{\boldsymbol{b}}\right)$ and $f$ is a gyromagnetic constant ( $\left[f B_{\text {ext }}\right]$ has units of frequency). In the absence of absorption, $\varepsilon$ and $f$ must be real. The polarizability can then be approximated as $\boldsymbol{\alpha} \sim \alpha_{I} \mathbf{I}+\boldsymbol{\alpha}_{\mathrm{MO}}$ [24], where $\alpha_{I}$ is the isotropic scalar polarizability found in the absence of a static magnetic field and $\boldsymbol{\alpha}_{\mathrm{MO}}$ is the antisymmetric MO tensor,

$$
\alpha_{I}=\left(\alpha_{0}^{-1}-\frac{i k^{3}}{6 \pi}\right)^{-1}, \quad \alpha_{0}=3 V \frac{\varepsilon-\varepsilon_{h}}{\varepsilon+2 \varepsilon_{h}},
$$

with

$$
\boldsymbol{\alpha}_{\mathrm{MO}}=\alpha_{\mathrm{MO}}\left(\begin{array}{ccc}
0 & -i b_{z} & i b_{y} \\
i b_{z} & 0 & -i b_{x} \\
-i b_{y} & i b_{x} & 0
\end{array}\right) \text {, }
$$

where $\alpha_{\mathrm{MO}} \equiv \frac{\alpha_{I}^{2}}{V} \frac{\gamma B_{\mathrm{ext}}}{\omega}$ and $\gamma=f(\omega) /\left(\varepsilon-\varepsilon_{h}\right)^{2}$.

In general, the time-averaged force on a dipolar nonisotropic particle can be obtained from the well-known expression

$$
\mathbf{F}=\frac{1}{2} \operatorname{Re}\left\{\sum_{j} p_{j} \nabla E_{j}^{*}\right\}=\frac{\varepsilon_{0} \varepsilon_{h}}{2} \operatorname{Re}\left\{\sum_{i, j} \alpha_{j i} E_{i} \nabla E_{j}^{*}\right\} .
$$

Splitting the polarizability in Hermitian and anti-Hermitian contributions,

$$
\frac{\mathbf{p}_{ \pm}}{\varepsilon_{0} \varepsilon_{h}} \equiv \frac{\boldsymbol{\alpha} \pm \boldsymbol{\alpha}^{\dagger}}{2} \mathbf{E}
$$

the force can be rewritten as the sum of "gradient" and "extinction" forces, $\mathbf{F} \equiv \mathbf{F}_{\text {grad }}+\mathbf{F}_{\text {ext }}$, with

$$
\begin{gathered}
\mathbf{F}_{\text {grad }}=\frac{1}{4} \nabla\left(\mathbf{E}^{*} \cdot \mathbf{p}_{+}\right), \\
\mathbf{F}_{\text {ext }}=-\frac{1}{2} \operatorname{Re}\left\{\left(\mathbf{E}^{*} \cdot \nabla\right) \mathbf{p}_{-}+\mathbf{E}^{*} \times\left(\nabla \times \mathbf{p}_{-}\right)\right\} .
\end{gathered}
$$

Equations (8) and (9) are general results for arbitrary nonisotropic electric dipolar particles showing that the force contribution from the Hermitian polarizability $\mathbf{F}_{\text {grad }}$ is always conservative while the anti-Hermitian contribution $\mathbf{F}_{\text {ext }}$ (which is not zero even in absence of absorption) can give rise to nonconservative optical forces.

In the particular case of a small MO response, the forces induced by the combination of external dynamic electric $\mathbf{E}$ and static magnetic $\mathbf{B}_{\text {ext }}$ fields can be obtained from Eqs. (8) and (9): The gradient force [Eq. (8)] is given by

$$
\begin{aligned}
\mathbf{F}_{\text {grad }}= & \nabla\left(\operatorname{Re}\left\{\alpha_{I}\right\} \frac{\varepsilon_{0} \varepsilon_{h}|\mathbf{E}|^{2}}{4}\right) \\
& +\frac{1}{2} \nabla\left(\operatorname{Re}\left\{\alpha_{\text {MO }}\right\} \omega \mathbf{J}_{\text {spin }} \cdot \hat{\boldsymbol{b}}\right),
\end{aligned}
$$

where the first term is the standard force proportional to the field intensity gradient and $\mathbf{J}_{\text {spin }}$ is the spin angular momentum (SAM) per unit volume of the light field.

The second MO term can be rewritten as a "Zeemann" force on an effective magnetic dipole of light $\mathbf{m}_{\text {eff }}$ in a external magnetic field $\mathbf{B}_{\text {ext }}$,

$$
\mathbf{F}_{\mathrm{grad}}^{\mathrm{MO}}=\nabla\left(\mathbf{m}_{\mathrm{eff}} \cdot \mathbf{B}_{\mathrm{ext}}\right),
$$

where the magnetic moment is proportional to the SAM density of the radiation, $\mathbf{m}_{\text {eff }} \equiv \gamma_{\text {eff }} \mathbf{J}_{\text {spin }}$, with a gyromagnetic ratio $\gamma_{\text {eff }}=\operatorname{Re}\left\{\alpha_{I}^{2} \gamma\right\} / 2 V$. When the external magnetic field is constant, there is a net MO force when $\mathbf{m}_{\text {eff }}$, i.e., the SAM density of the incident field, is not uniform,

$$
\mathbf{F}_{\text {grad }}^{\mathrm{MO}}=\left(\mathbf{B}_{\text {ext }} \cdot \nabla\right) \mathbf{m}_{\mathrm{eff}}+\mathbf{B}_{\text {ext }} \times\left(\boldsymbol{\nabla} \times \mathbf{m}_{\text {eff }}\right) .
$$

In contrast, since the SAM of a plane wave is uniform, there is no MO contribution to the gradient force. However, in the presence of an inhomogeneous static magnetic field, a gradient force on the particle exists, whose sign depends on the spin of light,

$$
\left.\mathbf{F}_{\text {grad }}^{\mathrm{MO}}\right|_{\mathrm{PW}}=\gamma_{\mathrm{eff}}\left(\mathbf{J}_{\mathrm{spin}} \cdot \nabla\right) \mathbf{B}_{\mathrm{ext}} \cdot
$$

If one of the components of $\mathbf{B}_{\text {ext }}$ is perpendicular to $\mathbf{k}$, with a gradient along the $k$ direction, there is a net $\mathrm{MO}$ force orthogonal to the isotropic contribution. This resembles the Stern-Gerlach effect of splitting a beam of atoms by a magnetic field gradient. However, here, the splitting does not depend on the magnetic moment of the particles but on the spin of the light field. So, for radiation with nonzero spin and for a nonconstant magnetic field, the direction to which the scattered light from the MO particle will be deflected is dependent on the spin angular momentum of the radiation, in full analogy with the well-known Stern-Gerlach experiment.

On the other hand, the extinction force [Eq. (9)] in the MO perturbative approach can be written as

$$
\mathbf{F}_{\mathrm{ext}}=k \operatorname{Im}\left\{\alpha_{I}\right\}\left(\frac{n_{h}}{c} \mathbf{S}-\frac{c}{2 n_{h}} \nabla \times \mathbf{J}_{\mathrm{spin}}\right)+\mathbf{F}_{\mathrm{ext}}^{\mathrm{MO}},
$$

where $\mathbf{S}=\operatorname{Re}\left(\mathbf{E}^{*} \times \mathbf{H}\right) / 2$ is the Poynting vector. Again, the first term is the known result for extinction forces on isotropic 

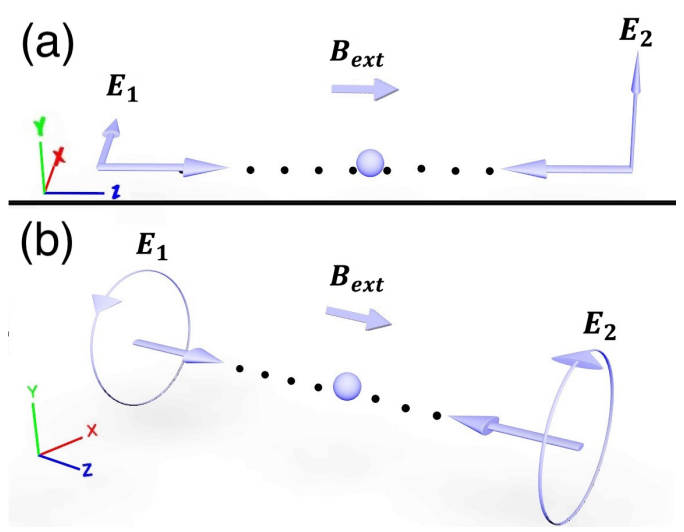

FIG. 1. (a) Electromagnetic field with $\phi=0$ consisting of two linearly cross-polarized plane waves. (b) Electromagnetic field with $\phi=\pi / 4$ consisting of two counterpropagating circularly polarized beams with the same helicity. The external constant magnetic field $\left(B_{\text {ext }}\right)$ is applied on the direction of propagation of the two beams.

particles [25] and, when the external magnetic field is constant,

$$
\begin{aligned}
\mathbf{F}_{\mathrm{ext}}^{\mathrm{MO}}=-\frac{\varepsilon_{0} \varepsilon_{h} \operatorname{Im}\left\{\alpha_{\mathrm{MO}}\right\}}{2} & \left(\operatorname{Re}\left\{\left(\mathbf{E}^{*} \cdot \nabla\right) \mathbf{E}\right\} \times \hat{\boldsymbol{b}}\right. \\
& \left.+\operatorname{Re}\left\{\mathbf{E}^{*} \times(\hat{\boldsymbol{b}} \cdot \nabla) \mathbf{E}\right\}\right) .
\end{aligned}
$$

Equations (11) and (15) are the main results of the present work concerning the MO contribution to the optical forces. For plane-wave (PW) illumination, $\mathbf{E}=\mathbf{E}_{0} e^{i \mathbf{k} \cdot \mathbf{r}}$, the MO extinction force is given by

$$
\left.\mathbf{F}_{\mathrm{ext}}^{\mathrm{MO}}\right|_{\mathrm{PW}}=k \operatorname{Im}\left\{\alpha_{\mathrm{MO}}\right\} \frac{n_{h}}{c} \frac{\operatorname{Im}\left\{\mathbf{E} \cdot \mathbf{H}^{*}\right\}}{2} \frac{(\mathbf{k} \cdot \hat{\boldsymbol{b}}) \mathbf{k}}{k^{2}},
$$

where $\operatorname{Im}\left\{\mathbf{E} \cdot \mathbf{H}^{*}\right\}$ is proportional to the optical helicity density $\Lambda$, defined as $[26,27]$

$$
\Lambda=\frac{n_{h}}{c} \frac{\varepsilon_{0} \varepsilon_{h}}{2 \omega} \operatorname{Re}\left\{\mathbf{E}^{*} \cdot \frac{\nabla \times \mathbf{E}}{k}\right\}=\left(\frac{n_{h}}{c}\right)^{2} \frac{\operatorname{Im}\left\{\mathbf{E} \cdot \mathbf{H}^{*}\right\}}{2 \omega} .
$$

The spin density has dimensions of helicity flux density (or angular momentum per unit volume) and for plane waves $\mathbf{J}_{\text {spin }}=\left(c / n_{h}\right) \Lambda \mathbf{k} / k$. Thus, we can write

$$
\left.\mathbf{F}_{\mathrm{ext}}^{M O}\right|_{\mathrm{PW}}=\operatorname{Im}\left\{\alpha_{\mathrm{MO}}\right\} \frac{\omega c}{n_{h}} \Lambda \frac{(\mathbf{k} \cdot \hat{\boldsymbol{b}}) \mathbf{k}}{k} .
$$

In general, the extinction force when $\mathbf{E} \cdot \hat{\boldsymbol{b}}=0$ and $\mathbf{E}$ varies only in the $\hat{\boldsymbol{b}}$ direction can be written as

$$
\mathbf{F}_{\mathrm{ext}}^{\mathrm{MO}} \cdot \hat{\boldsymbol{b}}=\operatorname{Im}\left\{\alpha_{\mathrm{MO}}\right\} \omega^{2} \Lambda .
$$

Note how the MO extinction force can be used to quantify the actual value of the helicity of the light field.

For plane-wave illumination and constant static magnetic field, the MO contributions above are usually small compared to the familiar isotropic contributions. In order to illustrate some of the unusual properties of magneto-optical forces we shall consider the field of a standing wave formed by two counterpropagating plane waves (see Fig. 1),

$$
\mathbf{E}=\frac{E_{0}}{\sqrt{2}}\left\{(\cos \phi \hat{\boldsymbol{x}}+i \sin \phi \hat{\boldsymbol{y}}) e^{i k z}+(i \sin \phi \hat{\boldsymbol{x}}+\cos \phi \hat{\boldsymbol{y}}) e^{-i k z}\right\},
$$
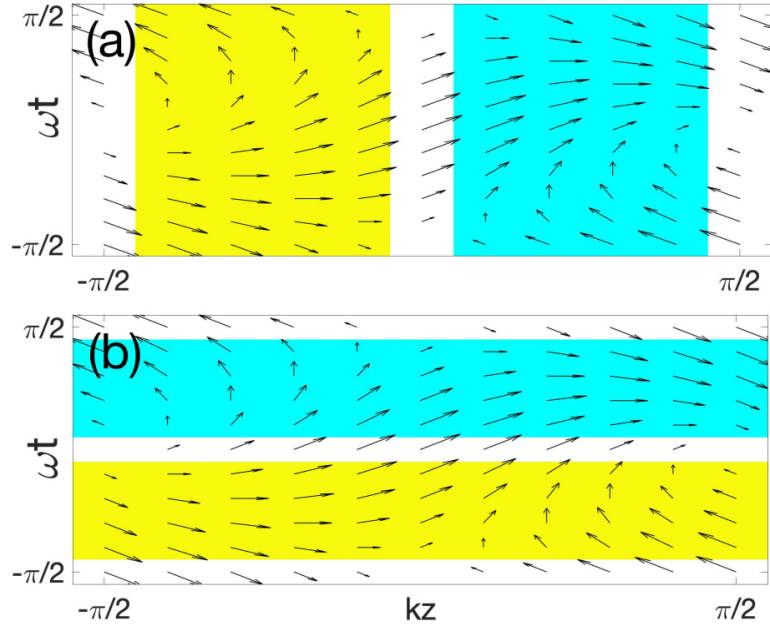

FIG. 2. Electric field vector vs position and time for two linearly cross-polarized plane waves, counterpropagating. In the color map we highlight the positive (yellow), zero (white), and negative (blue) values of (a) spin and (b) helicity.

with $\nabla|\mathbf{E}|^{2}=\mathbf{0}, \mathbf{S}=\mathbf{0}$, and $\boldsymbol{\nabla} \times \mathbf{J}_{\text {spin }}=\mathbf{0}$, i.e., a field that induces zero net force on an isotropic particle in the absence of a static magnetic field. Such optical fields had been utilized in the laser cooling of certain atoms [28] as well as in the discussion of the nature of the light spin $\left(\mathbf{J}_{\text {spin }}\right)$ and helicity $(\Lambda)$ densities of interfering waves [29] which are given by

$$
\begin{gathered}
\mathbf{J}_{\text {spin }}=-\frac{\varepsilon_{0} \varepsilon_{h}}{2 \omega}\left|E_{0}\right|^{2} \sin (2 k z)\left(\cos ^{2} \phi-\sin ^{2} \phi\right) \hat{z}, \\
\Lambda=\frac{n_{h}}{c} \frac{\varepsilon_{0} \varepsilon_{h}}{\omega}\left|E_{0}\right|^{2} \sin \phi \cos \phi .
\end{gathered}
$$

If $\mathbf{B}_{\text {ext }}=B_{\text {ext }} \hat{z}$ is a constant vector pointing along $z$ (in the so-called "polar" configuration), the total optical force acting on the MO particle is given by $\mathbf{F}=\mathbf{F}_{\text {grad }}^{\mathrm{MO}}+\mathbf{F}_{\mathrm{ext}}^{\mathrm{MO}}$, where $\mathbf{F}_{\text {grad }}^{\mathrm{MO}}$ is the spin (gradient) force [Eq. (11)] and $\mathbf{F}_{\mathrm{ext}}^{\mathrm{MO}}$ is an helicity (extinction) force [Eq. (16)],

$$
\mathbf{F}_{\text {grad }}^{\mathrm{MO}}=-\frac{\varepsilon_{0} \varepsilon_{h} k}{2}\left|E_{0}\right|^{2} \operatorname{Re}\left\{\alpha_{\mathrm{MO}}\right\}\left(\cos ^{2} \phi-\sin ^{2} \phi\right) \cos (2 k z) \hat{z},
$$

$$
\mathbf{F}_{\mathrm{ext}}^{\mathrm{MO}}=\varepsilon_{0} \varepsilon_{h} k\left|E_{0}\right|^{2} \operatorname{Im}\left\{\alpha_{\mathrm{MO}}\right\} \sin \phi \cos \phi \hat{z} .
$$

For the first setup with $\phi=0$, we have plotted, in Fig. 2, the electric field vector for different values of position and time. In Fig. 2(a) we have highlighted in a color map the corresponding different values of the spin. Note how, for any value of time, the spin oscillates in space from positive to negative values. This behavior gives rise to a gradient force coming from the classical "Zeeman" effect [Eq. (11)]. Although the incoming fields do not interfere, the scattered components generate a self-induced one-dimensional optical lattice emerging from the interference of the (magnetically induced) scattering components with the corresponding parallel components of the external beams. As shown in Fig. 2(b), for any position, the helicity oscillates in time from positive to negative values, meaning a null time-averaged value of the helicity and a zero extinction force [Eq. (19)]. In the second setup with $\phi=\pi / 4$ the situation is just the opposite. The 


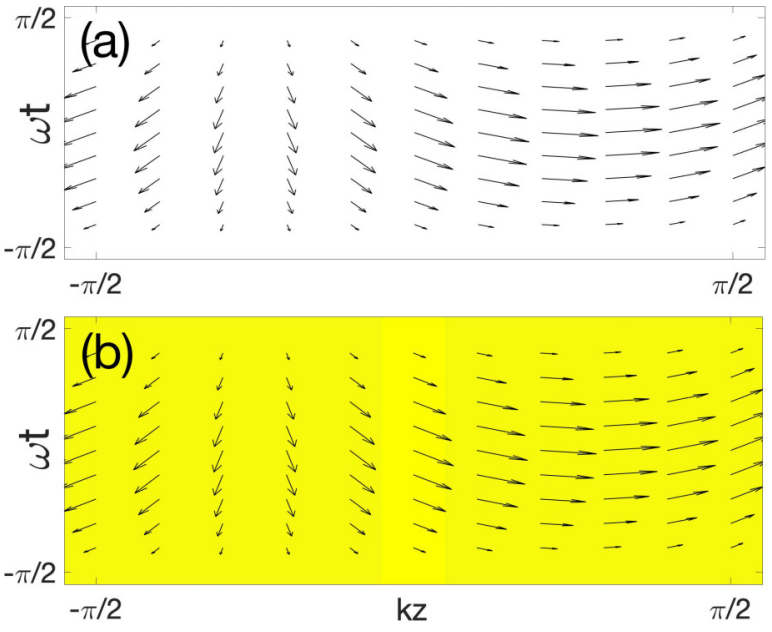

FIG. 3. Electric field vector vs position and time for two counterpropagating circularly polarized beams with the same helicity. In the color map we highlight the positive (yellow) and zero (white) values of (a) spin and (b) helicity.

total value of the spin is always equal to zero [note the white background in Fig. 3(a)], implying a null gradient force, while the helicity has a constant positive value [note the yellow background in Fig. 3(b)], implying the existence of a constant extinction force.

\section{OPTICAL TORQUE ON MAGNETO-OPTICAL PARTICLES}

In addition to optical forces, it is also possible to generate optical torques on electric dipoles. In the analysis of the MO-induced torque, it is useful to consider the total power dissipated by the particle [21],

$$
P_{\text {dis }}=\frac{\varepsilon_{0} \varepsilon_{h} \omega}{2} \mathbf{E}^{\dagger}\left(\frac{\boldsymbol{\alpha}-\boldsymbol{\alpha}^{\dagger}}{2 i}-\frac{k^{3}}{6 \pi} \boldsymbol{\alpha}^{\dagger} \boldsymbol{\alpha}\right) \mathbf{E},
$$

which vanishes in the absence of absorption, i.e., when $\boldsymbol{\alpha}_{0}=\boldsymbol{\alpha}_{0}^{\dagger}$.

When the particle absorbs radiation, we can obtain the absorption cross section which, defined for plane-wave incidence, depends on the helicity \pm 1 of the incoming field (we associate circularly left polarized light with positive helicity),

$$
\mathbf{E}_{\mathrm{PW}}=E_{0} \frac{\hat{\boldsymbol{x}} \pm i \hat{\boldsymbol{y}}}{\sqrt{2}} e^{i k z} .
$$

Assuming that we stay in the linear regime, and the magnetic field is parallel to the $k$ vector of the field $(\hat{\boldsymbol{b}} \cdot \mathbf{k}=k)$, we can easily obtain

$$
\sigma_{\mathrm{abs}}=\sigma_{\mathrm{abs}_{I}} \pm \sigma_{\mathrm{abs}_{\mathrm{dic}}}+O\left(B_{\mathrm{ext}}^{2}\right)
$$

with

$$
\sigma_{\mathrm{abs}_{I}}=k\left(\operatorname{Im}\left\{\alpha_{I}\right\}-\frac{k^{3}}{6 \pi}\left|\alpha_{I}\right|^{2}\right)
$$

and

$\sigma_{\mathrm{abs}_{\mathrm{dic}}}=\frac{\sigma_{\mathrm{abs}}^{+}-\sigma_{\mathrm{abs}}^{-}}{2}=k\left(\operatorname{Im}\left\{\alpha_{\mathrm{MO}}\right\}-\frac{k^{3}}{3 \pi} \operatorname{Re}\left\{\alpha_{I} \alpha_{\mathrm{MO}}^{*}\right\}\right)$, where $\sigma_{\mathrm{abs}_{I}}$ is the absorption cross section of the standard non-MO particle and $\sigma_{\mathrm{abs}_{\mathrm{dic}}}$ is the "dichroic" absorption cross section.

The general expression for the time-averaged torque $\boldsymbol{\tau}$ generated by an homogeneous wave on an electric dipole is given by [30]

$$
\boldsymbol{\tau}=\frac{1}{2} \operatorname{Re}\left\{\left(\mathbf{p} \times \mathbf{E}^{*}\right)-i \frac{k^{3}}{6 \pi \varepsilon_{0} \varepsilon_{h}} \mathbf{p} \times \mathbf{p}^{*}\right\},
$$

which, in the case of a MO particle, can be written as the sum of several contributions,

$$
\begin{aligned}
\boldsymbol{\tau}= & \frac{c}{n_{h}}\left(\sigma_{\mathrm{abs}_{I}} \mathbf{J}_{\text {spin }}\right)+\frac{c}{n_{h}} \sigma_{\mathrm{abs}_{\mathrm{dic}}} \frac{\varepsilon_{0} \varepsilon_{h}}{2 \omega}\left[|\mathbf{E}|^{2} \hat{\boldsymbol{b}}-\operatorname{Re}\left(\left(\hat{\boldsymbol{b}} \mathbf{E}^{*}\right) \mathbf{E}\right)\right] \\
& +\frac{c}{n_{h}}\left[k\left(\operatorname{Re}\left\{\alpha_{\mathrm{MO}}\right\}-\frac{k^{3}}{3 \pi} \operatorname{Im}\left\{\alpha_{I} \alpha_{\mathrm{MO}}^{*}\right\}\right)\right] \frac{\hat{\boldsymbol{b}} \times \mathbf{J}_{\text {spin }}}{2} \\
& +O\left(B_{\mathrm{ext}}^{2}\right) .
\end{aligned}
$$

For plane-wave illumination, with $\mathbf{k}=k \hat{\mathbf{z}}$, and in the "polar" configuration $\left(\mathbf{B}_{\mathrm{ext}}=B_{\mathrm{ext}} \hat{\mathbf{z}}\right)$, the torque is parallel to the $z$ axis and is given by

$$
\left.\boldsymbol{\tau}\right|_{\mathrm{PW}}=\frac{c}{n_{h}}\left[\sigma_{\mathrm{abs}_{l}} \mathbf{J}_{\mathrm{spin}}+\sigma_{\mathrm{abs}_{\mathrm{dic}}} \frac{\varepsilon_{0} \varepsilon_{h}}{2 \omega}|\mathbf{E}|^{2} \hat{\mathbf{z}}\right]+O\left(B_{\mathrm{ext}}^{2}\right) .
$$

Note how the torque generated by a plane wave has two components: a first well-known component, proportional to the average value of the spin density of the light field, and a second nonreported MO contribution, proportional to the squared modulus of the electromagnetic field. It means that a MO particle always experiences a torque (except in the nodes of a standing wave) independently of whether or not the electromagnetic field has a spin. The conservation of angular momentum is, however, fulfilled; compensation comes from the nonlinearly polarized scattered field and from the interference of the scattered and incoming fields [31]. Indeed, integration of the $z$ component of the angular momentum flux [32] of both fields (scattered and incident) over a surface of a sphere yields to torque compensation. Equation (32) demonstrates the remarkable counterintuitive result of a linearly polarized plane wave, carrying zero angular momentum, inducing a constant and permanent (as long as the magnetic field is applied) torque on a magneto-optically active nanoparticle.

\section{MAGNETO-OPTICAL VERSUS CHIRAL PARTICLES}

It is worth noticing that optical forces and torques on MO particles are qualitatively different from those on chiral objects. In particular, for the fields given by Eq. (20), the chiral forces and torques are equal to zero: The interaction force on a chiral electric dipole, neglecting self-interaction recoil terms, in a field with $\nabla|\mathbf{E}|^{2}=\mathbf{0}, \mathbf{S}=\mathbf{0}$, and $\nabla \times \mathbf{J}_{\text {spin }}=\mathbf{0}$, is given by [33-35]

$$
\boldsymbol{F}_{\mathrm{Ch}}=c \omega \operatorname{Re}(\chi) \nabla \Lambda+\frac{\omega^{2}}{c} \operatorname{Im}(\chi)\left(\mathbf{J}_{\mathrm{spin}}+\mathbf{J}_{\mathrm{spin}}^{\mathbf{m}}\right),
$$

with $\chi$ the chiral polarizability and $\mathbf{J}_{\text {spin }}^{\mathbf{m}}$ the magnetic spin of the electromagnetic radiation. For $\phi=0$, the force on a chiral particle is zero because both the helicity and the full spin vector (electric plus magnetic) are zero, while for $\phi=$ $\pi / 4$ the spin densities (electric and magnetic) are zero but the helicity is different from zero. However, the force on a chiral 
particle is again null because the helicity is constant and chiral particles are sensible only to variations of the helicity. On the other hand, the torque exerted by a plane wave on a chiral electric dipole, in linear response on $\chi$, is given by [36]

$$
\left.\boldsymbol{\tau}_{\mathrm{Ch}}\right|_{\mathrm{PW}}=c\left[\sigma_{\mathrm{abs}_{I}} \boldsymbol{J}_{\mathrm{spin}}+\sigma_{\mathrm{abs}_{\mathrm{dic}}}^{\mathrm{Ch}} \frac{1}{c \omega} \boldsymbol{S}\right]+O\left(\chi^{2}\right)
$$

with $\sigma_{\text {abs }}^{\text {Ch }}=2 k\left[\operatorname{Im}(\chi)-\frac{k^{3}}{6 \pi} \operatorname{Re}\left(\alpha_{I} \chi^{*}\right)\right]$ the "dichroic" absorption cross section of the chiral particle. Note that the MO torque depends on the intensity of the electric field, while in the case of a chiral torque, this term depends on the Poynting vector itself, which, for the fields given by Eq. (20), is zero.

\section{MAGNETO-OPTICAL FORCE ON $\boldsymbol{n}$-DOPED InSb PARTICLES}

As an example of a magneto-optical material we will calculate the values of the MO force for $n$-doped InSb, a polar semiconductor, that when subjected to an external magnetic field becomes magneto-optical [37]. The dielectric permittivity tensor can be nicely considered as a Drude-like metal which, at lowest order in the magnetic field, is given by [37]

$$
\begin{gathered}
\varepsilon=\varepsilon_{\infty}\left(1+\frac{\omega_{L}^{2}-\omega_{T}^{2}}{\omega_{T}^{2}-\omega^{2}-i \Gamma_{p} \omega}-\frac{\omega_{p}^{2}}{\omega\left(\omega+i \Gamma_{f}\right)}\right), \\
f(\omega)=\frac{\varepsilon_{\infty} \omega_{p}^{2}}{\left[\left(\omega+i \Gamma_{f}\right)^{2}\right]} \frac{e}{m^{*}} .
\end{gathered}
$$

Here, $\varepsilon_{\infty}$ is the high-frequency dielectric constant, $\omega_{L}$ is the longitudinal optical phonon frequency, $\omega_{T}$ is the transverse optical phonon frequency, $\omega_{p}^{2}=n_{p} e^{2} /\left(m^{*} \varepsilon_{0} \varepsilon_{\infty}\right)$ is the plasma frequency of free carriers of density $n_{p}$ and effective mass $m^{*}, \Gamma_{p}$ is the phonon damping constant, and $\Gamma_{f}$ is the freecarrier damping constant. In all the calculations below, we consider the particular case taken from Ref. [17], where $\varepsilon_{\infty}=$ 15.7, $\omega_{L}=36.2 \mathrm{THz}, \omega_{T}=33.9 \mathrm{THz}, \omega_{p}=31.4 \mathrm{THz}, \Gamma=$ $0.565 \mathrm{THz}, \gamma=3.39 \mathrm{THz}$, and $m^{*}=0.022 m_{0}$, and we use a 200-nm radius particle. In Fig. 4(a) we plot the maximum value of the optical force, in the $z$ direction, for the optical configuration with $\phi=0$ (force proportional to the gradient of the spin density of the light field) for different values of the frequency and the external magnetic field. Correspondingly, in Fig. 4(b) we plot again the value of the optical force but for the case with $\phi=\pi / 4$ (force proportional to the helicity). The force in the first case is proportional to the real part of $\alpha_{\mathrm{MO}}$, while in the second case it is proportional to the imaginary part of $\alpha_{\mathrm{MO}}$. The forces thus exhibit high values for the magnetically dependent branches of the resonances (see, e.g., Ref. [20]), which are zero for the nonmagnetic ones and, of course, for $B_{\text {ext }}=0$.

\section{CONCLUSIONS}

To sum up, we have introduced intriguing phenomena in optical forces caused solely by the presence of a sizable magneto-optical effect. We have demonstrated that the scattered fields from a MO-active nanoparticle allow for the generation of a conservative optical lattice using noninterfering incoming fields depending on the gradient of the spin density of the light field. This interaction allows for the proposal of
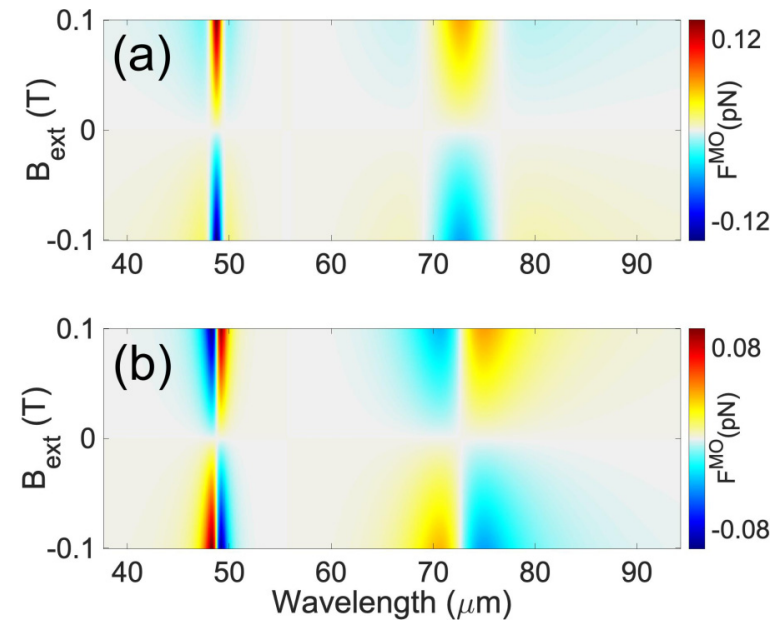

FIG. 4. Magneto-optical force vs wavelength and external magnetic field for (a) $\phi=0$ (two linearly cross-polarized plane waves, counterpropagating) and (b) $\phi=\pi / 4$ (two counterpropagating circularly polarized beams with the same helicity). The intensity of the electromagnetic field is $10 \mathrm{~mW} / \mu \mathrm{m}^{2}$.

a Stern-Gerlach experiment in which the scattered light is deflected on a nonconstant magnetic field, depending on the value of the spin angular momentum of the electromagnetic radiation. Also, we have calculated the extinction forces and we have shown how it is possible to exert tunable radiation pressure on a nanoparticle using electromagnetic fields with a zero average value of the Poynting vector but with constant helicity. Finally, we have proved that the MOE allows for a permanent nonreciprocal torque using a spinless, linearly polarized plane wave which, in contrast with the chiral case, depends not on the Poynting vector but on the intensity of the light field. These radiation forces and torques on nanoparticles based on the magneto-optical effect open a route to applications in the field of optical manipulation. On the one hand, this mechanism allows for a different interaction between light and a constant magnetic field. The spin properties of the electromagnetic radiation are now transferred to an effective magnetic dipole moment on a MO particle inducing a Zeemann interaction between the spin of light and the constant magnetic field. Inspired by this interaction, other applications can be proposed such as sorting of radiation based on a SternGerlach setup and cooling of nanosized particles lacking magnetic moment discrete energy levels. On the other hand, the magneto-optical interaction reported is also sensible to the value (not gradient) of the helicity of radiation and, for that reason, by using the MO particle as a probe, the absolute value of the helicity of a particular electromagnetic field can be determined. Finally, the magneto-optical effect also allows for the existence of optical manipulation schemes based on the nonreciprocal character of the MO interaction such as, for instance, the capability of transferring permanent torques in resonant cavities by using linearly polarized light.

\section{ACKNOWLEDGMENTS}

This research was supported by the Spanish MICINN and European Regional Development Fund (ERDF) through Projects No. FIS2015-69295-C3-1-P, No. 
FIS2015-69295-C3-3-P, No. PGC2018-095777-B-C21, No. PGC2018-095777-B-C22, the Basque Departamento de Educación through Project No. PI-2016-1-0041, and the María de Maeztu Program No. MDM-2014-0377.
[1] T. Sleator, T. Pfau, V. Balykin, O. Carnal, and J. Mlynek, Experimental Demonstration of the Optical Stern-Gerlach Effect, Phys. Rev. Lett. 68, 1996 (1992).

[2] R. P. Cameron, S. M. Barnett, and A. M. Yao, Discriminatory optical force for chiral molecules, New J. Phys. 16, 013020 (2014).

[3] N. Kravets, A. Aleksanyan, and E. Brasselet, Chiral Optical Stern-Gerlach Newtonian Experiment, Phys. Rev. Lett. 122, 024301 (2019).

[4] A. Karnieli and A. Arie, All-Optical Stern-Gerlach Effect, Phys. Rev. Lett. 120, 053901 (2018).

[5] O. Arteaga, E. García-Caurel, and R. Ossikovski, Stern-Gerlach experiment with light: Separating photons by spin with the method of A. Fresnel, Opt. Express 27, 4758 (2019).

[6] E. Arimondo, Coherent population trapping in laser spectroscopy, Prog. Opt. 35, 257 (1996).

[7] M. Fleischhauer and M. D. Lukin, Dark-State Polaritons in Electromagnetically Induced Transparency, Phys. Rev. Lett. 84, 5094 (2000).

[8] L. Karpa and M. Weitz, A Stern-Gerlach experiment for slow light, Nat. Phys. 2, 332 (2006).

[9] Y. Guo, L. Zhou, L.-M. Kuang, and C. P. Sun, Magneto-optical Stern-Gerlach effect in an atomic ensemble, Phys. Rev. A 78, 013833 (2008).

[10] C. Hang and G. Huang, Stern-Gerlach effect of weak-light ultraslow vector solitons, Phys. Rev. A 86, 043809 (2012).

[11] A. Ashkin, Optical trapping and manipulation of neutral particles using lasers, Proc. Natl. Acad. Sci. USA 94, 4853 (1997).

[12] V. V. Temnov, G. Armelles, U. Woggon, D. Guzatov, A. Cebollada, A. García-Martín, J.-M. García-Martín, T. Thomay, A. Leitenstorfer, and R. Bratschitsch, Active magnetoplasmonics in hybrid metal-ferromagnet structures, Nat. Photonics 4, 107 (2010).

[13] G. Armelles, A. Cebollada, A. García-Martín, and M. U. González, Magnetoplasmonics: Combining magnetic and plasmonic functionalities, Adv. Opt. Mater. 1, 10 (2013).

[14] G. Armelles, B. Caballero, A. Cebollada, A. Garcia-Martin, and D. Meneses-Rodríguez, Magnetic field modification of optical magnetic dipoles, Nano Lett. 15, 2045 (2015).

[15] M. Rollinger et al., Light localization and magneto-optic enhancement in Ni antidot arrays, Nano Lett. 16, 2432 (2016).

[16] N. de Sousa, L. S. Froufe-Pérez, J. J. Sáenz, and A. GarcíaMartín, Magneto-optical activity in high index dielectric nanoantennas, Sci. Rep. 6, 30803 (2016).

[17] E. Moncada-Villa, V. Fernández-Hurtado, F. J. García-Vidal, A. García-Martín, and J. C. Cuevas, Magnetic field control of nearfield radiative heat transfer and the realization of highly tunable hyperbolic thermal emitters, Phys. Rev. B 92, 125418 (2015).

[18] P. Ben-Abdallah, Photon Thermal Hall Effect, Phys. Rev. Lett. 116, 084301 (2016)
[19] R. M. Abraham Ekeroth, A. García-Martín, and J. C. Cuevas, Thermal discrete dipole approximation for the description of thermal emission and radiative heat transfer of magneto-optical systems, Phys. Rev. B 95, 235428 (2017).

[20] R. M. A. Ekeroth, P. Ben-Abdallah, J. C. Cuevas, and A. GarcíaMartín, Anisotropic thermal magnetoresistance for an active control of radiative heat transfer, ACS Photonics 5, 705 (2018).

[21] S. Albaladejo et al., Radiative corrections to the polarizability tensor of an electrically small anisotropic dielectric particle, Opt. Express 18, 3556 (2010).

[22] R. P. Hunt, Magneto-optic scattering from thin solid films, J. Appl. Phys. 38, 1652 (1967).

[23] L. D. Landau, E. Lifshitz, and L. Pitaevskii, Electrodynamics of Continuous Media (Pergamon, Oxford, UK, 1984), Vol. 8.

[24] H. Marinchio, J. J. Sáenz, and R. Carminati, Light scattering by a magneto-optical nanoparticle in front of a flat surface: Perturbative approach, Phys. Rev. B 85, 245425 (2012).

[25] S. Albaladejo, M. I. Marqués, M. Laroche, and J. J. Sáenz, Scattering Forces from the Curl of the Spin Angular Momentum of a Light Field, Phys. Rev. Lett. 102, 113602 (2009).

[26] R. P. Cameron, S. M. Barnett, and A. M. Yao, Optical helicity, optical spin and related quantities in electromagnetic theory, New J. Phys. 14, 053050 (2012).

[27] M. Nieto-Vesperinas, Optical theorem for the conservation of electromagnetic helicity: Significance for molecular energy transfer and enantiomeric discrimination by circular dichroism, Phys. Rev. A 92, 023813 (2015).

[28] J. Dalibard and C. Cohen-Tannoudji, Laser cooling below the Doppler limit by polarization gradients: Simple theoretical models, J. Opt. Soc. Am. B 6, 2023 (1989).

[29] R. P. Cameron, S. M. Barnett, and A. M. Yao, Optical helicity of interfering waves, J. Mod. Opt. 61, 25 (2014).

[30] M. Nieto-Vesperinas, Optical torque on small bi-isotropic particles, Opt. Lett. 40, 3021 (2015).

[31] M. Mansuripur, Energy, linear momentum, and angular momentum exchange between an electromagnetic wave-packet and a small particle, J. Nanophotonics 13, 012503 (2018).

[32] S. M. Barnett, Optical angular-momentum flux, J. Opt. B 4, S7 (2001).

[33] A. Canaguier-Durand, J. A. Hutchison, C. Genet, and T. W Ebbesen, Mechanical separation of chiral dipoles by chiral light, New. J. Phys. 15, 123037 (2013).

[34] S. B. Wang and C. T. Chan, Lateral optical force on chiral particles near a surface, Nat. Commun. 5, 3307 (2014).

[35] A. Hayat, J. P. Balthasar Mueller, and F. Capasso, Lateral chirality-sorting optical forces, Proc. Natl. Acad. Sci. USA 112 13190 (2015).

[36] H. Chen et al., Optical torque on small chiral particles in generic optical fields, Opt. Express 25, 32867 (2017).

[37] E. Palik et al., Coupled surface magnetoplasmon-optic-phonon polariton modes on InSb, Phys. Rev. B 13, 2497 (1976). 